\title{
Complex Robot Assembly Production Line of New Energy Component
}

\author{
Yi Du, Feng Shi and Xikun Lu \\ Anyang institute of technology, Anyang, China
}

\begin{abstract}
To analyze the capacity configuration problem of flexible assembly line under the environment of engineering-to-order with resource-oriented, an optimal model with dual constraints which are system production capacity (throughput) and order due-date (makespan) was built to minimize the equipment investment costs. Because the optimal problem is a random nonlinear integer programming problem, firstly, based on the model of open queuing network with finite buffers, an expansion method was developed to obtain the system performance measurers, and the relationship between buffer allocation and capacity configuration was presented. Then a heuristic optimization algorithm was put forward to determine the optimal program of capacity configuration. Finally, experiments to assess the accuracy of the expansion method were reported by comparing the results with simulation, and a case was studied to obtain the reasonable optimal program of capacity configuration.
\end{abstract}

Keywords-queuing network; flexible assembly line; capacity configuration; heuristic algorithm

\section{INTRODUCTION}

With the implementation of intelligent manufacturing strategy, the robot flexible manufacturing cell is increasingly used by manufacturing companies. Pointed on the problems of single and small batch, customizing production in mould manufacturing, the robot flexible manufacturing cell need real-time scheduling to accommodate the changing of the external environment as well as changes in the type of mould parts, operation and processing time of orders. The theory and method of flexible manufacturing cell have not yet been well suited for solving such problems currently. For example, firstly, if the parts, machines or robots change, then the structure and size of model changes, so the modeling work is cumbersome and complex. Secondly, the cell has many parts, whose processing time are short and delivery is urgent, and flexible transporting requirements cause competition confusion for the multiple robots on the single track. Thirdly, there is no simulation and evaluation system, which contains the function of deadlock control and corrective plan.

\section{QUESTIONS}

Reviews of flexibility showed approaches for examining the flexibility of cell-line.however the effect of cell-line is still unknown.The simulation of manufacturing systems as an active area of research.some researches have compared cell-line and FS in a simulation study .Others have used simulation to study flexible manufacturing systems (FMS) Another category of research is using simulation to understand the limitations of CM systems. Ikou Kaku[1] used a simulation model of an actual FS to compare cell-line with traditional FS. The products are grouped by ranked order cluster algorithm into three groups. The results of the experiment showed the cell-line system (including two workers; three workers; six workers) to be inferior in minimization of total throughput time and the total labor hours.

Bagher and Bashiri(2014) Considering the personnel assigned, production unit layout, on the basis of production units and production unit in the lowest cost of minimum distances between parts, equipment reconstruction and operator minimum matching problem as the goal, build the model establish nonlinear programming production unit; Under the uncertain demand of the market environment, how to configure the limited resources, to bring the best gains or rapid response to market demand, to be a key issue in the enterprise decisions need to be solved. With the increase of product uncertainty demand, many scholars have studied the uncertain demand of products. Koenigsberg points out that the research on the problem of production line don't get enough attention, he put forward in the process of production line design and operation has three main problems: one is the number of workstations in production line, the second is the location of the cache area, three is the capacity of the cache area, there are currently no tools to solve these problems. Hou wenhao et al. described the man-machine ratio model in multi-unit flexible manufacturing system and established a model to solve the complex interference system performance. The results show that the man-machine interference in the production system has a significant influence on the performance of the system. Yi Du(2015) analysis of the solution of variable production line reconstruction model. According to the results of the analysis, the unified model is solved by two steps and then combined. The first step is to obtain all the resource allocation results that may obtain the optimal solution. Including the number of production lines and the resource allocation scheme, the multi-channel resource allocation method with the "cell" multi-channel cutting is not possible to obtain the optimal solution. The second step is to study the scheduling algorithm, and to solve the scheduling problem by using traditional optimization algorithm, improved optimization algorithm and improved particle swarm optimization algorithm for order characteristics and user requirements. And compare the optimization of the algorithm.

From the literature reviews of manufacturing system design, only a few papers were found that added integrating the design strategies for robust manufacturing system one disadvantage of their model is that the planning horizon is only for equipment. 
Another disadvantage of their model is that the planning horizon is only for a single period.

Abdelmila[10]optimized the productivity of CM systems using a two-stage model .A good heuristic should be provided to achieve shorter computational time in soloing large scale provlems.

\section{PROBLEM DESCRIPTION}

The model meets the following assumptions:

(1) The random process of arrival system of different types of workpiece is poisson process. The arrival rate is $\lambda_{1}$ and $\lambda_{2}$;

(2) The service timeof nodes at all levels is subject to negative exponential distribution, the average machining rate of each manipulator is $\mu_{i}(i=1,2)$ The average processing rate of each moving robot is, $\mu_{i}(i=3,4)$;

(3) All levels node contains number of machine is $c_{i}(1,2,3,4)$, Ordinary machines can only process one piece at a time, and two workpieces on the assembly machine are machined at the same time, and the processing process cannot be interrupted once it is started.

Set a certain buffer capacity, with an average productivity system (the system output per unit time is the average number of finished product) for constrained flexible assembly line production equipment configuration optimization problem, the goal is to optimize the equipment configuration so as to minimize the investment cost, satisfy the requirement of system capacity and order delivery date.

The definition parameters are as follows:

$p_{i}$ : the unit price of the mechanical arm of the production line, $\mathrm{i}=1,2$;

$q_{i}$ : The unit price of the i robot, $\mathrm{i}=1,2$;

$x_{i}$ : The number of the i manipulator configurations, $\mathrm{i}=1,2$;

$y_{i}$ : The number of the i robot configuration, $\mathrm{i}=1,2$;

$\Theta$ : The system average output rate ;

Based on the assumptions of the system model, the mathematical model of the optimization problem is as follows:

$$
\min P=p_{i} x_{i}+q_{i} y_{i}
$$

s.t.

$$
\begin{array}{r}
\Theta\left(x_{i}, y_{i}\right)>\alpha \\
p_{i}, q_{i}, x_{i}, y_{i} \in N^{+}
\end{array}
$$

Among them, formula (1) represents the optimization goal, which is to minimize the cost of equipment investment;
Equation (2) represents the average capacity constraint, The actual average output rate $\Theta(\boldsymbol{X}, \boldsymbol{Y})$ not less than the target average output rate $\alpha$; Formula (3) represents $p_{i}, q_{i}, x_{i}, y_{i}$ nonnegative integer.

\section{AlgORITHM}

\section{A. Network Reconfiguration}

Assembly station, two artifacts on the equipment for processing at the same time, to solve the problem of the constraints, this paper will stand assembly into two conventional workstation with work at the same time constraints, by assembling node split, the two workpiece assembly simultaneity constraint into two child nodes of the workpiece at the same time to carry on the processing and completed at the same time and leave.

\section{B. Parameter Estimation}

By queuing theory $\mathrm{M} / \mathrm{M} / \mathrm{c} / \mathrm{K}$ model theory is available, There are c service Windows in the system, and the system capacity is $\mathrm{K}$, The customer arrives at the system at the strength $\lambda$ of the poisson flow., The independent service time of each window is subject to the negative exponential distribution of strength ${ }^{\mu}$, The steady-state formula of the system parameters is as follows:

The probability of system idle:

$$
\begin{aligned}
& \text { when } \rho=\lambda /(c \mu) \neq 1, \quad p_{0}=\left[\sum_{n=0}^{c-1} \frac{(c \rho)^{n}}{n !}+\frac{(c \rho)^{c}}{c !} \frac{1-\rho^{K-c+1}}{1-\rho}\right]^{-1} \text {; when } \\
& \rho=1, \quad p_{0}=\left[\sum_{n=0}^{c-1} \frac{c^{n}}{n !}+\frac{c^{c}}{c !}(K-c+1)\right]^{-1} .
\end{aligned}
$$

The probability of loss of the system: $P_{K}=\frac{c^{c} \rho^{K}}{c !} p_{0}$.

Average waiting time:

$$
\begin{aligned}
& \text { when } \begin{array}{l}
\rho \neq 1 \\
W_{q}=\frac{c^{c} \rho^{c+1} p_{0}}{c !(1-\rho)^{2}}\left[1-(K-c+1) \rho^{K-c}+(K-c) \rho^{K-c+1}\right] / \lambda_{e}
\end{array} \\
& \text { when } \rho=1, \quad W_{q}=\frac{c^{c}}{2 c !}(K-c)(K-c+1) p_{0} / \lambda_{e} .
\end{aligned}
$$

\section{THE EXAmple ANALYSIS}

The operating system environment of the calculation example is Microsoft win7, the hardware environment is CPU $1.86 \mathrm{GHz}, 2.00 \mathrm{~GB}$ RAM, and Matlab 2010b is adopted for the software platform of the analytical algorithm. 
TABLE I. THE EXAMPLE PARAMETER

$\begin{array}{cccccc}\lambda_{1} & \lambda_{2} & \mu_{1} & \mu_{2} & \mu_{11} & \mu_{22} \\ 0.9 & 0.9 & 0.3 & 0.3 & 0.3 & 0.3 \\ 0.9 & 0.9 & 0.3 & 0.3 & 0.3 & 0.3 \\ 0.9 & 0.9 & 0.3 & 0.3 & 0.3 & 0.3 \\ 0.9 & 0.9 & 0.3 & 0.3 & 0.3 & 0.3 \\ 0.9 & 0.9 & 0.3 & 0.3 & 0.3 & 0.3 \\ c_{1} & c_{2} & c_{11} & c_{22} & \Theta & \\ 4 & 2 & 2 & 2 & 0.456 & \\ 4 & 2+1 & 2 & 2 & 0.631 & \\ 4 & 2+2 & 2 & 2 & 0.834 & \\ 4 & 2 & 2 & 2-1 & 0.471 & \\ 4 & 2+2 & 2 & 3 & 0.835 & \end{array}$

In the face of current production requirements such as short life cycle, unfixed types, mass batches, and small quantity for each batch, the paper establishes a nonlinear integer model. Its objective function is tardiness, and its constraints are equipment cost and human resources cost. As tardiness is the results of resource distribution and product scheduling, and as scheduling is complex and diverse, the paper proposes a two-phase model, applies the branch and bound algorithm to simplify dimensions, and provides the scheme of POSP-FM product scheduling to obtain the optimal solution under the scheme.

The research herein factors out multi-skill labors. During actual production, it is important to configure multi-skill labors with regard to optimization of production lines. Consideration is possibly given to research on a split model of variable production line with multi-skill labors in the future.

\section{REFERENCES}

[1] IKou Kaku A Mathematical Model for Converting Conveyor Assembly Line to Cellular Manufacturing IEMS Vol.7. No.2.pp.160-170, Sepertember 2008.

[2] Bagheri,M.,Bashiri,M.,(2014a).A new mathematical model towards the integration of cell formation with operator assignment and inter-cell layout problems in a dynamic environment. Applied Mathematical Modelling,38(4),1237-1254.

[3] Bonfietti,A.Lombardi,M.,Benini, Li, Milano,M.(2014).CROSS cyclic resource-constrained scheduling solve. Artificial Intelligence,206(1),25-52.

[4] Yi Du, Qing-xin Chen,Ning Mao.The model and simulation of SMT production[J]. Advanced materials research, 2012.

[5] Aldaihani, M.M.(2015).Scheduling methodologies for a flexible manufacturing cell with non-identical parallel machines and a robot.International Journal of Industrial \&Systems Engineering 21(4),499-514.

[6] Katsuhide Isa \& Tsuyoshi Tsuru.(2002). Cell Production Innovation in Japan: toward a New Model for Japanese Manufacturing[J]. Industrial Relations, 41(4): 548-578.

[7] Li Xiag, Chen Qingxin \& MAO Ning. (2014). Buffer allocation optimization of flow shop based on queueing network model[J]. Computer Integrate Manufacturing System, 20(4): 890-897 (in Chinese).

[8] Liu Chengang, Stecke, E. \& Lian Jie. (2012). Reconfiguration of assembly systems: from conveyou assembly line to serus. Journal of Manufacturing Systems, 31(3): 312-325.
[9] Meller, R.D. (1998). White paper on multi-channel manufacturing. Technical Report Department of Industrial and Systems Engineering [J]. 42(5): 19-20.

[10] Siemia Tkowski \& Mprzybylski, W. (2007). Modelling and simulation analysis of process alternatives in the cellular manufacturing of axially symmetric parts [J]. International Journal of Advanced Manufacturing Technology, Vol. 32, No. 5-6, pp. 516-530.

[11] Steche,K.E.,Yin,Y.,Kaku,I.\& Murase, Y.(2012). Seru:the organizational extension of JIT for a super-talent factory.International Journal of Strategic Decision Sciences,3(1),105-108 